\title{
Regulation effects of total flavonoids in Morus alba L. on hepatic cholesterol disorders in orotic acid induced NAFLD rats
}

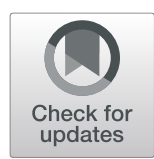

Yucheng $\mathrm{Hu}^{1 \dagger}$, Jingqi $\mathrm{Xu}^{2 \dagger}$, Qian Chen ${ }^{1}$, Mengyang Liu², Sijian Wang ${ }^{1}$, Haiyang Yu' , Yi Zhang ${ }^{2^{*}}$ and Tao Wang ${ }^{1 *}$

\begin{abstract}
Background: Mulberry leaves are the dried leaves of Morus alba L., flavonoids from mulberry leaves (MLF) has showed regulatory effect on abnormal lipid metabolism, but the regulatory mechanism of MLF on cholesterol metabolism is still missing. This study was designed to investigate the effect of MLF and its active metabolite quercetin on regulating cholesterol disorders.

Methods: The mechanism of MLF on alleviating liver injury and regulating cholesterol was examined in dyslipidemic SD rats. The regulatory mechanism of quercetin for cholesterol disorders have also been detected through lipid laden HepG2 cell model.

Results: Our results showed that MLF significantly inhibited lipid accumulation and alleviate hepatic injury in NAFL $D$ rat model. The hepatic expression level of SREBP2, HMGCR and miR-33a were significantly down-regulated, while CYP7A1 was induced by MLF treatment. In vitro, Quercetin significantly decreased lipid accumulation in HepG2 cells. Mechanistically, quercetin could inhibit the mRNA and protein expression level of SREBP2 and HMGCR with or without LDL treatment. In addition, quercetin could also reduce the LXRß while induced SR-BI mRNA expression.

Conclusion: Our findings indicate that MLF and quercetin could reduce the excessive cholesterol accumulation in vivo and in vitro. These cholesterol-regulating phenomenon might attribute to its effect on down-regulating the expression of lipid-related markers such as SREBP2 and HMGCR, which may exert a protective role in the NAFLD treatment.
\end{abstract}

Keywords: Mulberry leaves flavonoids, Quercetin, Cholesterol metabolism, NAFLD

\section{Background}

Nonalcoholic fatty liver disease (NAFLD) is a common chronic disease characterized by excessive accumulation of lipids in the liver other than alcohol intake [1]. Although NAFLD is not fatal, it is considered as main risk factors of liver cancer and cardiovascular disease (CVD), such as hypertension, arteriosclerosis and coronary heart

\footnotetext{
* Correspondence: zhwwxzh@263.net; wangtao@tjutcm.edu.cn

${ }^{\dagger}$ Yucheng Hu and Jingqi Xu contributed equally to this work.

${ }^{2}$ Tianjin State Key Laboratory of Modern Chinese Medicine, Tianjin University

of Traditional Chinese Medicine, Tianjin 300193, China

${ }^{1}$ Institute of Traditional Chinese Medicine, Tianjin University of Traditional

Chinese Medicine, Tianjin 300193, China
}

disease [2]. At present, the correlation between NAFLD and CVD is still unclear, but the results indicate that lipid accumulation is considered as the important mediator between the two risks [3]. A previous meta-analysis showed that NAFLD patients had a $64 \%$ higher risk of fatal and/or non-fatal CVD events than non-NAFLD patients [3].

Elevated serum cholesterol and low-density lipoprotein-cholesterol (LDL-C) levels are the main triggers of NAFLD caused CVD $[4,5]$. Under normal condition, synthesis of cholesterol is tightly regulated by sterol regulatory element binding protein 2 (SREBP2) and its

(c) The Author(s). 2020 Open Access This article is licensed under a Creative Commons Attribution 4.0 International License, which permits use, sharing, adaptation, distribution and reproduction in any medium or format, as long as you give appropriate credit to the original author(s) and the source, provide a link to the Creative Commons licence, and indicate if changes were made. The images or other third party material in this article are included in the article's Creative Commons licence, unless indicated otherwise in a credit line to the material. If material is not included in the article's Creative Commons licence and your intended use is not permitted by statutory regulation or exceeds the permitted use, you will need to obtain permission directly from the copyright holder. To view a copy of this licence, visit http://creativecommons.org/licenses/by/4.0/ The Creative Commons Public Domain Dedication waiver (http://creativecommons.org/publicdomain/zero/1.0/) applies to the data made available in this article, unless otherwise stated in a credit line to the data. 
down-stream cholesterol synthesis enzymes such as HMG-CoA reductase (HMGCR) [6, 7]. Nearly 60\% synthesized cholesterol is transported from liver to plasma and plays an important role in keeping membrane structure and synthesis of hormones, and nearly $40 \%$ cholesterol is converted to bile acid by cholesterol-7 $\alpha$-hydroxylase (CYP7A1) [8]. Under the state of NAFLD, excessive lipid accumulation leads to steatosis in liver, and causes the up-regulation of SREBP2 and HMGCR, which results in cholesterol over-production [9]. At the same time, the increase of cholesterol content in the liver will reduce the conversion rate of bile acid and reduce cholesterol intake from the serum. These changes can further disrupt cholesterol homeostasis and increase the risk of CVD.

Natural products have been used to treat various kinds of diseases, owing to its anti-tumor, anti-inflammatory, antioxidant and hepatoprotective properties [10-12]. Due to the low toxicity and side effects of natural drugs, $40 \%$ of FDA-approved treatment are natural ingredients or derivatives $[12,13]$.

As a life style related disease, there is no specific medication to treat moderate NAFLD in clinic. Dietary supplements or complementary alternatives are becoming a choice for prevention and therapy of NAFLD [14]. And inhibition of NAFLD through the natural products have been extensively studied and reported [15].

Mulberry leaves are the dried leaves of $M$. alba L., which is used as the medicinal and edible plants in south-east Asia for a long period [16]. The chemical constituents of mulberry leaves contain flavonoids (quercetin and its homologue), alkaloids (deoxynojirimycin), sitosterols and polysaccharides. Within them, quercetin and its homologue are the major constituents and the content is more than $1 \%$ [17]. It has been reported that mulberry leaves have the pharmacological effect of attenuating hepatic steatosis and insulin resistance in high-fat diet-fed mice [18]. And its active compound quercetin could reduce postprandial blood glucose, lowering blood lipid, anti-inflammation and anti-tumor [19]. In addition, quercetin was also used to regulate hepatic lipid metabolism in traditional clinic [20]. Recently, it was reported that mulberry leaves extract showed significant effect on reducing plasma cholesterol level in rats induced by high-fat diet, but the mechanism of action is not clear [18].

In this study, we investigated the mechanism of the flavonoids of mulberry leaves (MLF) on cholesterol regulation in dyslipidemic rats. In addition, the role of quercetin, main metabolites of flavonoids in MLF, in cholesterol disorders was also determined in lipid laden HepG2 cell model, and the mechanism of its action was further explored.

\section{Methods}

\section{Plant material}

The mulberry leaves were collected from Dezhou county, Shandong province, China, and identified by Dr. Yi Zhang as leaves of $M$. alba L. The voucher specimen was deposited at the Academy of Traditional Chinese Medicine of Tianjin University of Traditional Chinese Medicine (TJUTCM; No.2013083101).

Methanol extract of the leaves of M. alba L. was provided by the Chinese Medicine Chemistry Laboratory of TJUTCM, stored at the room. In brief, the mulberry leaves were ground $(500 \mathrm{~g})$ and extracted with methanol (5 L) under reflux for two times. The combined extracting solution was concentrated in vacuum to afford a methanol extract. Quercetin and rutin content in methanol extract were 0.9 and $2.6 \%$ determined by HPLC method described previously [21]. Quercetin standard sample was obtained from Shanghai yuanye BioTechnology Co., Ltd.

\section{Animals and treatment}

Healthy male Sprague-Dawley (SD) rats (100-120 g, 4 weeks old) were purchased from Vital River Laboratory Animal Technology Co., Ltd., (Beijing, China). All rats had a free access to water and standard chow diets and were housed in cages with temperature-controlled (20$26^{\circ} \mathrm{C}$ ) for $12 \mathrm{~h}$ of light/dark cycles. After a week of adaptation, the rats were divided into six groups $(n=10$ per group), including normal group (Normal: a balanced diet), control group (Control: 1\% OA diets with 33\% sucrose content), fenofibrate group (Feno: OA diets with oral administration of $\left.50 \mathrm{mg} \mathrm{kg}^{-1} \mathrm{~d}^{-1}\right)$, and methanol extract of mulberry leaves groups (MLF: OA diets with oral administration of MLF 200, 100 and $50 \mathrm{mg} \mathrm{kg}^{-1}$ $\left.\mathrm{d}^{-1}\right)$. The maximum oral administration doses for rat $\left(200 \mathrm{mg} \mathrm{kg}^{-1} \mathrm{~d}^{-1}\right)$ was translated from the traditional human daily dose for fatty liver disease and extraction yield rate. The test samples were dissolved in solution of $5 \%$ gum arabic and administered orally to rats via gavage. Orotic acid (OA) and uratan were obtained from Shanghai Future Industry Co, Ltd. and Shanghai Ekear Co, Ltd. respectively. All other reagents were purchased from Sigma-Aldrich except where indicated. All animal procedures were performed in compliance with the Guidance Suggestions for the Care and Use of Laboratory Animals issued by the Ministry of Science and Technology of China (2006) and Science and Technological Committee and the Animal Use and Care Committee of TJUTCM (No. 201810009).

\section{Determination of hepatic lipid content}

The rats were fasted for $12 \mathrm{~h}$ at the end of the experiment. The rats were anesthetized by intraperitoneal injection of $20 \%$ uratan with a dose of $1 \mathrm{ml} / 100 \mathrm{~g}$ (body 
weight). After rats dissected, the blood was pumped out of the liver by D-Hanks perfusion through portal vein, and the liver was removed and rinsed with pre-cooled normal saline, and then the rats were sacrificed by cervical dislocation. Liver total lipid content was extracted as previously described with slight modifications [22]. The commercial assay kits were used to detect the contents of total cholesterol (TC) and liver triglyceride (TG) in the liver.

\section{Hepatic histological evaluation}

The liver samples were fixed in $4 \%$ paraformaldehyde, dehydrated in ethanol gradient solutions and embedded in paraffin wax for hematoxylin-eosin (H\&E) staining. The liver frozen sections were stained with Oil Red O as previously described [23]. And an Axio Imager D2 microscope (Zeiss, Oberkochen, Germany) was applied to take photographs for histological and morphological analysis. H\&E-stained liver sections were assessed blindly for NAFLD activity score (NAS) and lobular inflammation [24, 25]. The number of fat drops and fat distribution in liver tissues and hepatocytes were observed. The following scoring criteria were used to score the steatosis of liver tissues. If the distribution of lipid droplets in hepatocytes is rare, it is denoted as 0 . No more than $25 \%$ of the area will be counted as 1 point. No more than $50 \%$ is counted as 2 points, no more than $75 \%$ as 3 points, and almost all of the lipid droplets account for 4 points. The scoring criteria of hepatic lobular inflammation were as follows. If no necrosis is seen, it is denoted as 0. A small amount of inflammatory cell infiltration and necrosis was counted as 1 point. Moderate inflammatory cell infiltration was recorded as 2 points and massive necrosis was recorded as 3 points.

\section{Immunohistochemistry assay}

To determine SREBP2, HMGCR and CYP7A1 expression, the liver sections were permeabilized with $0.5 \%$ (v/v) Triton X-100 for $10 \mathrm{~min}$, blocked with $2 \%$ bovine serum albumin (BSA) for $2 \mathrm{~h}$ at $\mathrm{RT}$, and then incubated with SREBP2 (ab112046, Abcam Plc., UK), HMGCR (ab174830, Abcam) and CYP7A1 (EL907377-100, EterLife) primary antibody respectively overnight at $4{ }^{\circ} \mathrm{C}$. After removal of the primary antibody by washing with PBS, the sections were incubated with biotin conjugated goat anti-rabbit IgG for $15 \mathrm{~min}$ at RT. After washing with PBS, the sections were incubated in an HRP-conjugated avidin solution for $20 \mathrm{~min}$ followed by adding the developing solution. The images of cross sections were viewed and photographed by Axio Imager D2 microscope (Zeiss, Oberkochen, Germany).
Cell culture and cell viability assay

HepG2 cells (SCSP-510) was purchased from the Chinese Cell Culture Center (Shanghai, China) and cultured in Eagle's minimal essential medium (MEM), with $10 \%$ fetal bovine serum (FBS) and $1 \%$ penicillin and streptomycin and $1 \%$ non-essential amino acids (NEAA) and incubated at $37{ }^{\circ} \mathrm{C}$ in a humidified atmosphere of $5 \% \mathrm{CO} 2$.

To determine the cell viability, HepG2 cells were seeded at $5 \times 10^{3}$ cells/well into 96-well plates for $24 \mathrm{~h}$, treated with quercetin at indicated concentrations $(1,5$, $10,30,60,90,150$, and $\left.180 \mu \mathrm{moll}^{-1}\right)$ for $24 \mathrm{~h}$ and reincubated with MTT solution at $37{ }^{\circ} \mathrm{C}$ for $4 \mathrm{~h}$. Then the medium was removed and added $150 \mu \mathrm{l}$ DMSO to each well, oscillated for $10 \mathrm{~min}$. The absorbance was measured at $490 \mathrm{~nm}$.

\section{Analysis of cholesterol in HepG2 cells}

A cholesterol accumulation model of HepG2 cells caused by LDL was established. $2 \times 10^{5}$ cells/well were seeded in 48-well plates. After $24 \mathrm{~h}$, the cells were incubated without FBS for $1 \mathrm{~h}$, and then were treated without (control) or with $25 \mu \mathrm{g} \mathrm{ml}^{-1} \mathrm{LDL}$. The final concentrations of quercetin (Que; 30, 10 and $5 \mu \mathrm{mol}^{-1}$ ) and LDL + Que. (quercetin 30,10 $\mu \mathrm{mol} \mathrm{l}^{-1}$ ) were used as the treatment group. Hepatocytes were harvested for analysis of TC and protein.

\section{Real-time quantitative PCR analysis}

RNA isolation, cDNA synthesis and real-time PCR analysis were performed as described previously [22]. Both human and rat gene primer sequences used for real-time PCR were shown in Table 1 . Results were presented as levels of expression relative to those of controls after normalization to GADPH using the $2^{-\triangle \triangle C T}$ methods. Analysis was carried out in triplicates.

\section{Determination of SREBP2 and HMGCR protein by immunofluorescent staining}

HepG2 cells were seeded on cover slips in 24-well plates and treated with quercetin at indicated concentrations. After $24 \mathrm{~h}$, the supernatant was removed and washed with PBS 3 times and then fixed with $4 \%$ paraformaldehyde for $30 \mathrm{~min}$. After removal of paraformaldehyde by aspiration and washing with PBS, cells were blocked with $2 \%$ BSA for $2 \mathrm{~h}$, and then incubated with SREBP2 or HMGCR antibodies overnight at $4{ }^{\circ} \mathrm{C}$. Cells were reincubated with the secondary antibody for $1 \mathrm{~h}$ at room temperature. After washing with PBS, the slips were stained with DAPI solution for nuclei. The slips were observed by using Axio Imager D2 (Zeiss, Oberkochen, Germany), and the images of cells were photographed. 
Table 1 Primer sequences used for real-time PCR analysis

\begin{tabular}{|c|c|c|}
\hline Gene & Forward & Reverse \\
\hline h-SREBP2 & AAGTCTGGCGTTCTGAGGAA & AGGTCCACCTCATTGTCCAC \\
\hline h-HMGCR & CTTGTGTGTCCTTGGTATTAGAGCTT & GCTGAGCTGCCAAATTGGA \\
\hline h-SR-BI & CTTAAGAACGTGCGCATCGA & TGCTITTGTGCCTGAACTCC \\
\hline h-LXRa & GGAGGTACAACCCTGGGAGT & AGCAATGAGCAAGGCAAACT \\
\hline$h-L X R \beta$ & ССССТТСТТСТТАССССАСТ & CGACTGTGACTGTGACTCCT \\
\hline h- $\beta$-actin & CTGGAACGGTGAAGGTGACA & AAGGGACTTCCTGTAACAATGCA \\
\hline r-SREBP2 & AGAAGGAGAAAGGCGGACAA & TCTCCTGGCGCAGTTTATGA \\
\hline$r-H M G C R$ & AGAGCTGGCTTGAAACACCTGA & TTTGGACTGGAGACGGATGTAGA \\
\hline r-CYP7A1 & TTGGAATAAGGAGAAGGAAAGC & GGAGTTTGTGATGAAATGGACA \\
\hline r-miR-33a & CCTCATAAGCGGTGCATTGTA & TATGCTTGTTCTCGTCTCTGTGTC \\
\hline r-GAPDH & GGTGAAGGTCGGTGTGAACG & CTCGCTCCTGGAAGATGGTG \\
\hline
\end{tabular}

SREBP2 sterol regulatory element binding protein 2; HMGCR HMG-CoA reductase; CYP7A1 cytochrome P450 family 7 subfamily A member 1 ; LXRa liver $X$ receptor a; LXR $\beta$ liver $X$ receptor $\beta$; SR-BI scavenger receptor class B type 1; GAPDH glyceraldehyde-3-phosphate dehydrogenase; miR-33a microRNA-33a

\section{Western blot analysis}

After treatment, total cellular proteins were extracted from cells followed by determination of SREBP2, HMGC $\mathrm{R}$ and $\beta$-actin (ab8227, Abcam) protein expression by Western blot as described [26]. Bands were visualized with a ChemiDoc MP Imaging System (Bio-Rad, Hercules, CA, USA), and the density were quantitated based on three repeated experiments.

\section{Statistical analysis}

All experiments were repeated three times. The results were analyzed using the SPSS 22.0 statistical software. The values were expressed as the mean \pm SEM. Sample comparison was evaluated by one-way ANOVA and LSD or Dunnett's T3 multiple comparison tests, and a $p$-value $<0.05$ was considered statistically significant.

\section{Results}

\section{MLF inhibited hepatic lipid accumulation in OA diet} induced rats

We initially determined the hepatic lipid content and found that TG level was significantly increased in OA fed control rats. MLF and fenofibrate treatment could significantly decrease the TG and TC levels in the liver (Fig. 1a and b).

To further determine the role of MLF, we assessed the hepatic histological and morphological changes by oil red $\mathrm{O}$ and $\mathrm{H} \& \mathrm{E}$ staining. Figure $1 \mathrm{c}$ demonstrated that there was significant hepatocyte hypertrophy and vacuolization with obvious hepatocyte damage caused by excessive lipid accumulation. However, fenofibrate and MLF treatment could reduce lipid droplets accumulation and alleviated the histological lesion in rat liver fed with OA. Notedly, the liver tissue morphology of the MLF200 group was similar to that of Feno-50 group (Fig. 1c).
MLF improved hepatic cholesterol metabolism in OA diet induced rats

To further illustrate the potential mechanism of MLF on cholesterol metabolism, we assessed the expression levels of SREBP2, HMGCR and CYP7A1. As is shown in Fig. 2a and b, the hepatic SREBP2 and HMGCR expression were significantly induced in OA fed rats. Treatment of MLF and fenofibrate could decrease the expression of SREBP2 and HMGCR. Furthermore, treatment of MLF at different concentrations markedly up-regulated CYP7A1 mRNA expression, while downregulated its inhibitor-miR-33a (Fig. 2c and d). In addition, similar results were obtained by immunohistochemistry assay, suggesting MLF could also regulate cholesterol metabolism genes in protein level (Fig. 2e).

Quercetin inhibited hepatic cholesterol accumulation via regulating SREBP2 and HMGCR expression in HepG2 cells To further explore the mechanism of quercetin in regulating cholesterol metabolism, HepG2 cells were treated with quercetin at different concentrations. It was found that quercetin had no inhibitory effect on HepG2 cells activity at a concentration of $1-60 \mu \mathrm{M}$ (Fig. 3a). As Fig. $3 \mathrm{~b}$ showed, quercetin treatment could significantly reduce the lipid droplets accumulation in HepG2 cells, especially at dose of $30 \mu \mathrm{M}$. Consistently, total cholesterol also decreased with concentration increasing, and most significantly at $12 \mathrm{~h}$ (Fig. 3c).

Next, the expression levels of SREBP2 and HMGCR were detected. As shown in Fig. 3d-e and Fig. 4a-c, the mRNA and protein levels of SREBP2 and HMGCR were decreased by quercetin. In addition, the inhibitory effect of SREBP2 and HMGCR expression by quercetin was further confirmed by immunofluorescent staining (Fig. $4 d)$. These results imply that the cholesterol accumulation inhibition role of quercetin is related to reduction 
A

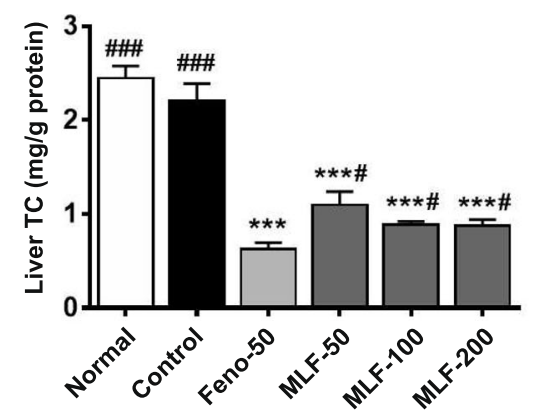

B

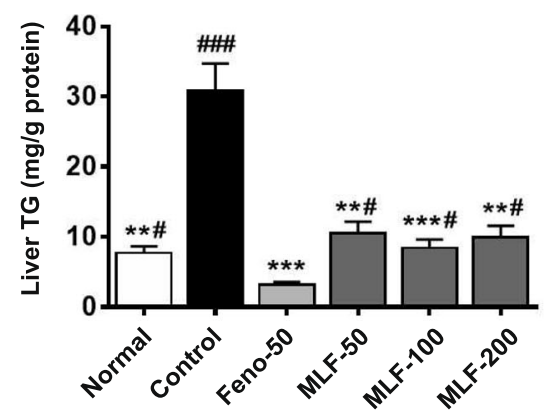

C

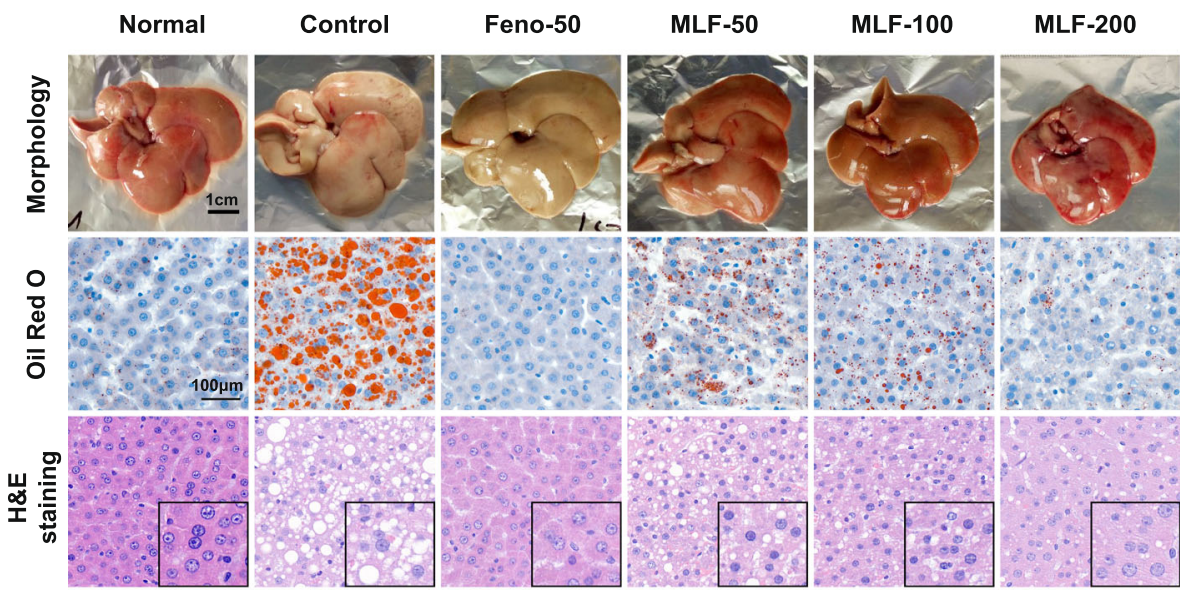

D

Overall NAS

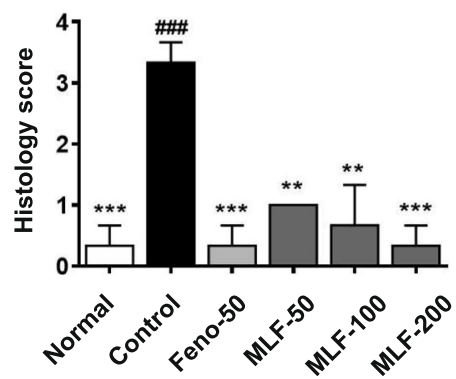

$\mathrm{E}$

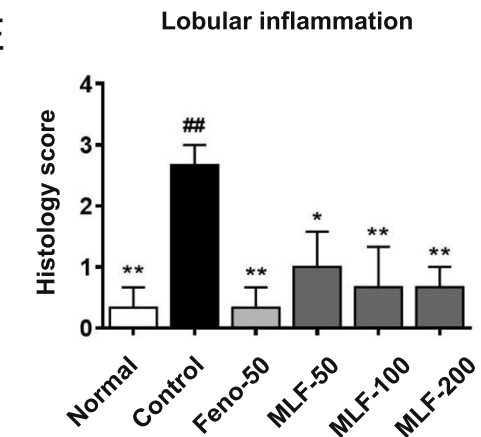

Fig. 1 Regulation effects of MLF on hepatic lipid accumulation in NAFLD rats. $\mathbf{a}$ and $\mathbf{b}$ TG and TC levels in the liver with or without oral administration of MLF at 200, 100 and $50 \mathrm{mg} \mathrm{kg}^{-1} \mathrm{~d}^{-1}$. c Morphology, hematoxylin-eosin (H\&E) staining and oil red O staining of liver tissues. Pictures were captured under a microscope (400x). $\mathbf{d}$ and $\mathbf{e}$. H\&E-stained liver sections were assessed for the NAFLD activity score (NAS) and lobular inflammation. Normal: normal group; Control: control group; Feno-50: positive control group (50 mg kg ${ }^{-1}$ ); MLF: mulberry leaves extract in different dosage groups. Data were presented as mean \pm SEM. $n=10,{ }^{*} p<0.05,{ }^{* *} p<0.01,{ }^{* * *} p<0.001$ vs. control group, \#p $<0.05, \# \#<0.01$, $\# \# \# p<0.001$ vs. Feno-50 group

of cholesterol synthesis, particularly through regulating SREBP2 and HMGCR expression.

\section{Quercetin inhibited LDL induced cholesterol accumulation} in HepG2 cells

Quercetin is reported to be the major metabolite of mulberry flavonoids after intestinal flora and hepatic metabolism in vivo [18]. Therefore, to further define the role of MLF, the effect of quercetin on LDL-induced cholesterol accumulation was tested in HepG2 cells. As shown in Fig. 5a, quercetin had no inhibitory effect on cells viability at concentration $1-90 \mu \mathrm{M}$. The oil red O staining results showed that quercetin treatment could significantly inhibit LDL-induced lipid accumulation in 
A

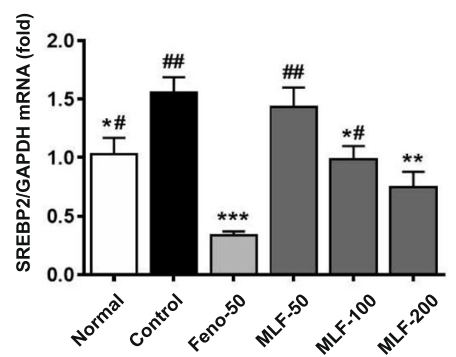

C

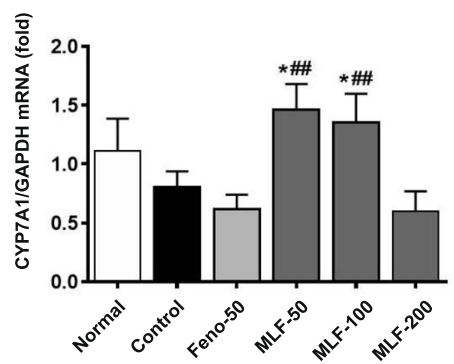

$E$
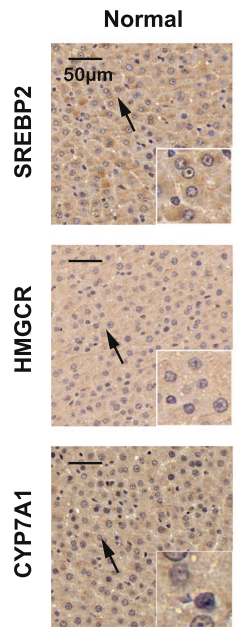

Control
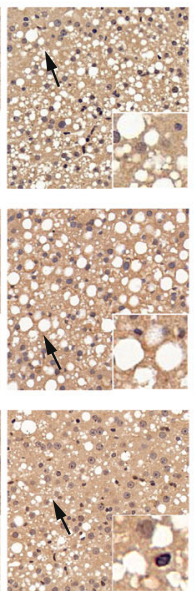

Feno-50
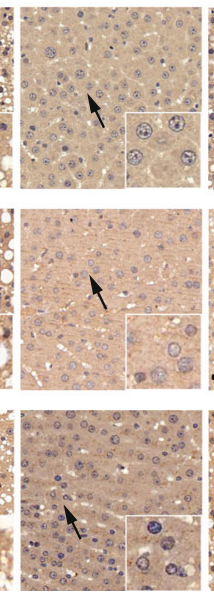

B

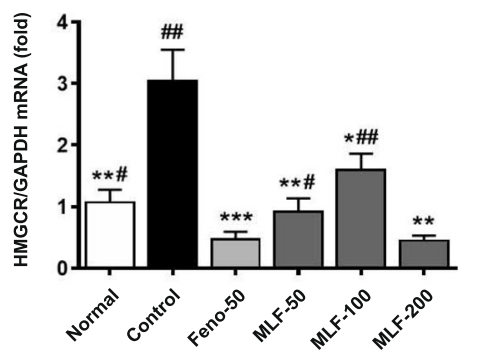

D

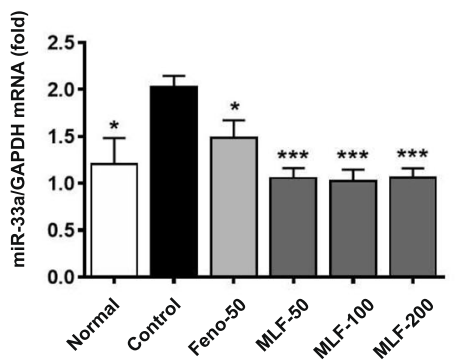

Fig. 2 The effect on the regulation of cholesterol disorders of MLF. a-d Expression of SREBP2, HMGCR, CYP7A1, miR-33a mRNA was analyzed by quantitative real-time PCR. e Expression of SREBP2, HMGCR, CYP7A1 protein were analyzed by immunohistochemistry assay. Normal: normal group; Control: control group; Feno-50: positive control group $\left(50 \mathrm{mg} \mathrm{kg}^{-1}\right)$; MLF: mulberry leaves extract in different dosage groups. Data were presented as mean \pm SEM. $n=10,{ }^{*} p<0.05,{ }^{* *} p<0.01,{ }^{* * *} p<0.001$ vs. control group, \#p $<0.05$, \#\#p $<0.01$, \#\#\#p $<0.001$ vs. Feno-50 group

HepG2 cells and gradually decrease the lipid droplets as the dose increased (Fig. 5b). Furthermore, we analyzed the total cholesterol levels in LDL-induced HepG2 cells after quercetin treatment. The results showed that after LDL induction, TC content was significantly stimulated, while the TC level of HepG2 cells decreased most significantly after the intervention of quercetin at $12 \mathrm{~h}$ (Fig. 5c).

\section{Quercetin regulated hepatic lipid related genes} expression in LDL-induced cholesterol laden HepG2 cells To further determine the potential mechanism of quercetin in cholesterol metabolism, cells were treated with
LDL or LDL with quercetin at different concentrations. The results showed that quercetin could significantly down-regulate the mRNA expression of SREBP2 and HMGCR (Fig. 5d and e). In addition, scavenger receptor class B type 1 (SR-BI) mRNA expression was induced by quercetin treatment (Fig. 5f). Interestingly, quercetin could significantly decrease the mRNA expression of LXR $\beta$ in LDL-induced HepG2 cells, while had no significant effect on LXR $\alpha$ (Fig. 5g and h). Consistent with the mRNA results, quercetin treatment could markedly reduce the SREBP2 and HMGCR protein expression in LDL induced cells (Fig. 6a-6d). 


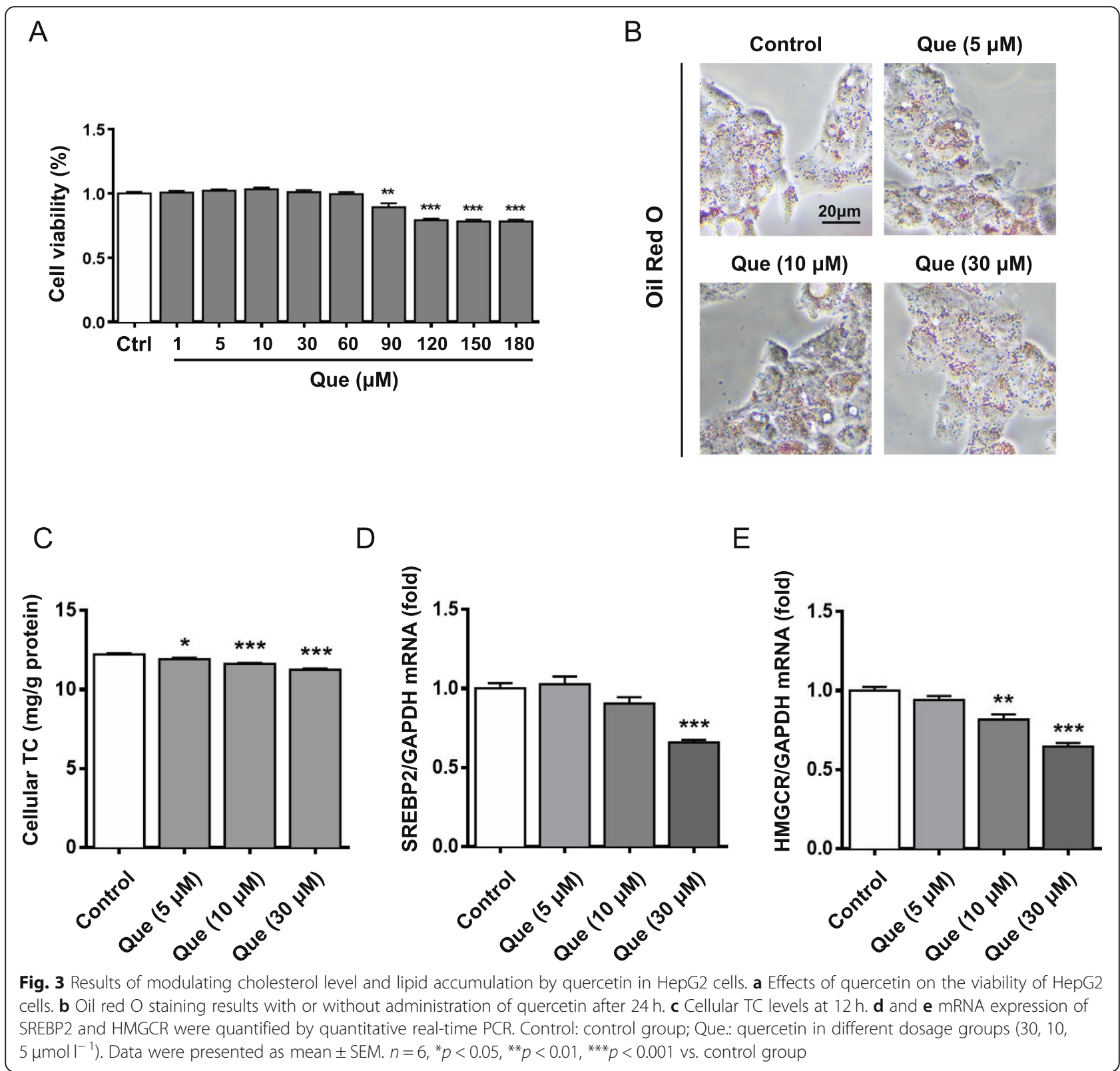

\section{Discussion}

NAFLD is a most common metabolic disease, which can gradually lead to series of disease, including liver cancer and cardiovascular disease. Initial stage of NAFLD is mild and often developing slowly [5]. Clinic research indicated that early treatment or lifestyle modification significantly reduced the incidence of complications. Although the causes of NAFLD is multi-factorial and not entirely clarified, excessive TG and cholesterol induced pathological changes in liver is considered as an important risk factor on NAFLD occurrence and development [27].

Under the state of NAFLD, major pathological changes are excessive lipid accumulation induced liver steatosis, tissue damage and necrosis [9]. The major pathophysiological changes include up-regulation of SREBP2, HMGCR, down-regulation of CYP7A1. SREBP2 and HMGCR are closely related to cholesterol synthesis in mammal liver. Furthermore, nearly $40 \%$ of cholesterol is converted to bile acids by CYP7A1. miR-33a is involved in the regulation of metabolic disorders, and specifically inhibits CYP7A1 mRNA [28]. These changes resulted in increasing of hepatic cholesterol content, decreasing conversion rate of cholesterol into bile acids, reducing uptake rate of cholesterol from the serum. Finally, it could cause destruction of cholesterol homeostasis and increasing the risk of CVD [29]. 

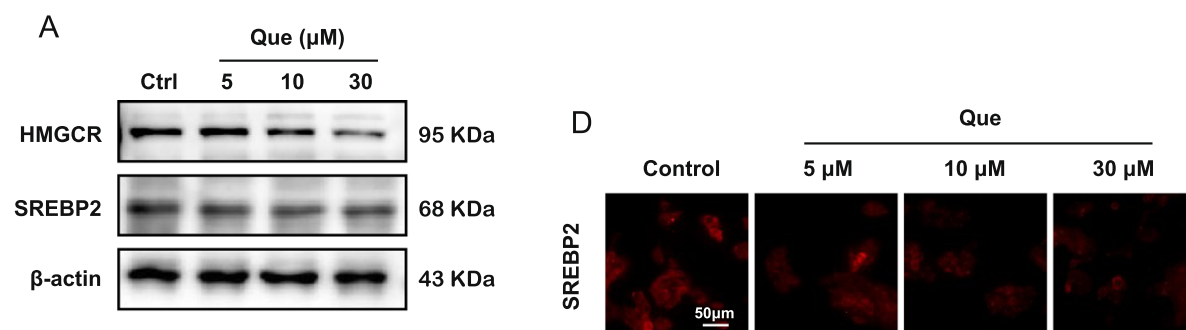

B
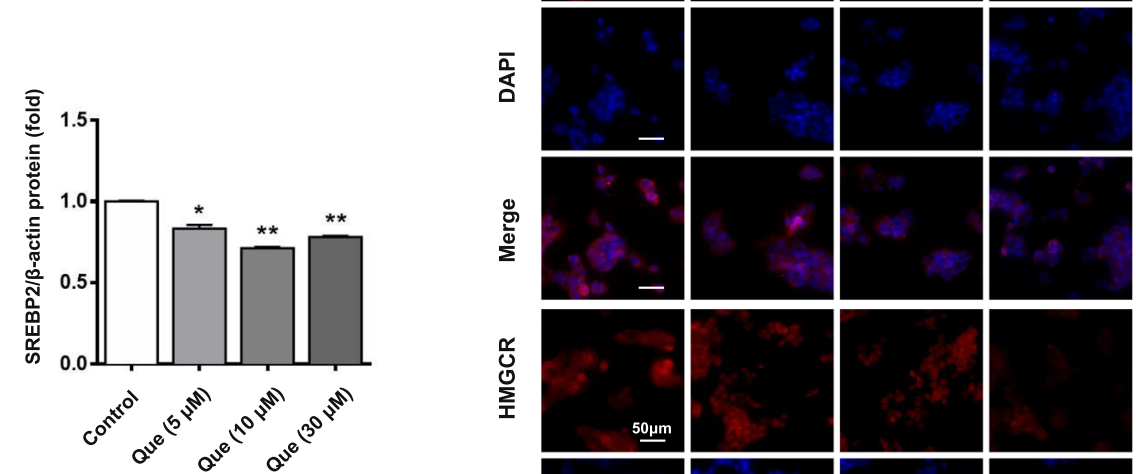

C
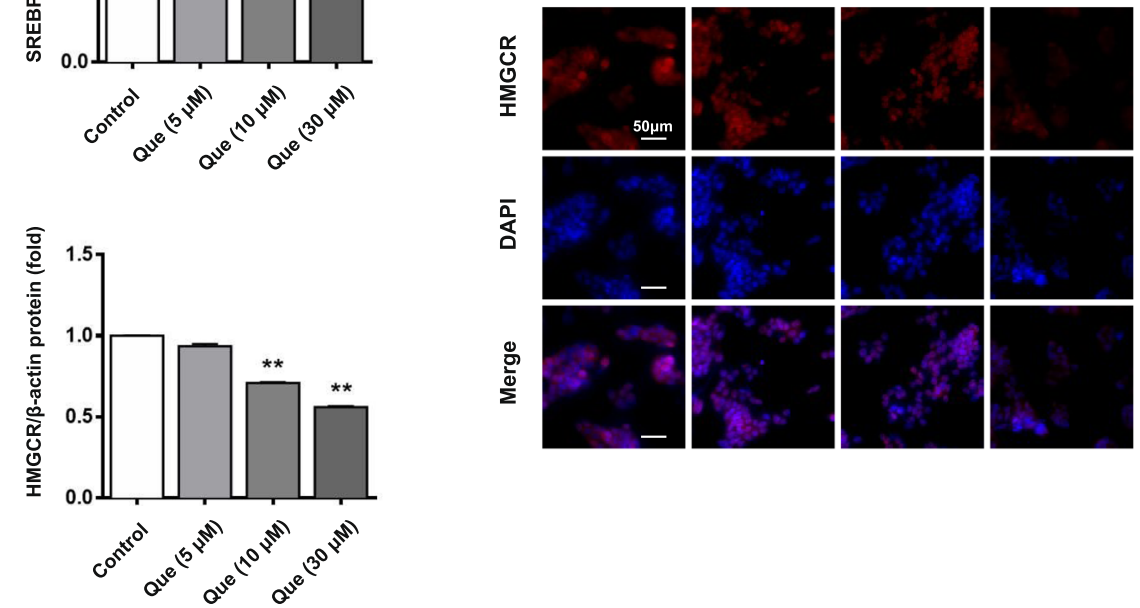

Fig. 4 The effect on regulating cholesterol disorders and mechanism of quercetin on the HepG2 cells. a-c Expression of SREBP2 and HMGCR protein were analyzed by western blotting. $\mathbf{d}$ Expression of SREBP2, HMGCR protein were analyzed by immunofluorescence assay. Control: control group; Que.: quercetin in different dosage groups $\left(30,10,5 \mu \mathrm{mol} \mathrm{I}^{-1}\right)$. Data were presented as mean $\pm \mathrm{SEM} . \mathrm{n}=6,{ }^{*} p<0.05,{ }^{*} p<0.01$ vs. control group

Currently, there are no specific drugs approved for NAFLD treatment. Cholesterol-lowering drugs, such as statins, have been used to treat NAFLD in clinic. However, there are also some inevitable side effects [30]. Recently, dietary supplements are becoming an option for the prevention and treatment of NAFLD. As a medicinal and edible plants resource, MLF has been proved to be useful for preventing the development of NAFLD [31]. Recent studies have confirmed that treatment with MLF for 4 weeks can improve the lipid status of patients with hypercholesterolemia, decrease serum TG, LDL and TC/ HDL ratios, and normalize the serum LDL level [32]. While the mechanism studies are still lacking, which limit its clinic use.

As previously reported [14], OA can induce NAFLD via promote fat deposition in the liver. In this study, we investigated the regulatory mechanism of MLF on cholesterol metabolism in OA-induced rats. It implied that the prevention of NAFLD by MLF in HFD induced rats may be attributed to inhibiting excessive production of cholesterol. Our results showed that MLF could significantly reduce the TC level accompanied by the upregulation of CYP7A1 expression in liver. In addition, we found that the high dose MLF $(200 \mathrm{mg} / \mathrm{kg}$ ) showed comparable lipid-lower effect of fenofibrate without causing adverse effect in this rat model.

The biosynthesis of cholesterol begins with taking acetyl-coenzyme A as the raw material and generates mevalonic acid pathway through the action of HMGCR and other enzymes. It is the speed limit and irreversible step of cholesterol synthesis. Therefore, HMGCR is of great medical significance which is strictly regulated by the nuclear transcription factor SREBP2. In our results, the expression level of SREBP2 and HMGCR were down-regulated, which inhibited the biosynthesis of cholesterol and further reduced the cholesterol accumulation in liver. Similarly, previous reports have shown that MLF significantly reduced blood glucose, 

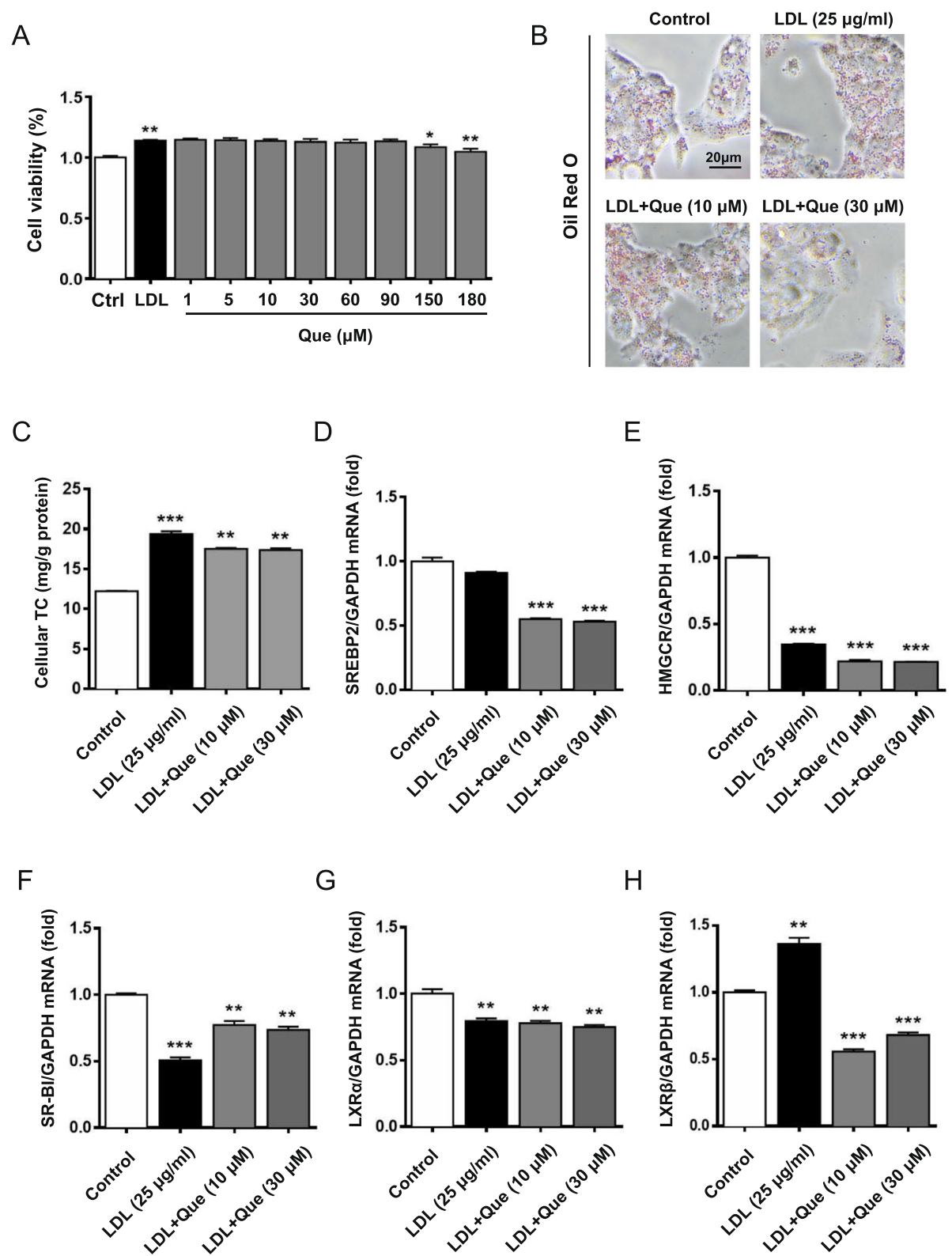

Fig. 5 Results of modulating cholesterol level and lipid accumulation by quercetin in LDL-induced cholesterol laden HepG2 cells. a Effects of quercetin on the viability of HepG2 cells in LDL model. b Oil red $\mathrm{O}$ staining results with or without administration of quercetin after $24 \mathrm{~h}$. $\mathbf{c}$ Cellular TC levels at 12 h. d-h mRNA expression of SREBP2, HMGCR, CYP7A1, SR-BI, LXRa and LXRß. Control: control group; LDL: a high cholesterol level simulation model of HepG2 cells by low density lipoprotein $\left(25 \mathrm{gg} \mathrm{ml}^{-1}\right)$; LDL + Que.: quercetin with LDL model HepG2 cells (30, $10 \mu \mathrm{mol}$ $\left.I^{-1}\right)$. Data were presented as mean \pm SEM. $n=6,{ }^{*} p<0.05,{ }^{* *} p<0.01,{ }^{* *} p<0.001$ vs. control group

serum TC, LDL-c and TG, and inhibit excessive accumulation of hepatic lipids in mice induced by high-fat diet [33, 34].

Quercetin, as one of the main flavonoids in mulberry leaves, is reported to be the major metabolite of mulberry flavonoids in vivo [18]. it exerts the functions of modulating cholesterol level and anti-inflammation [35, 36]. By using LDL induced cholesterol loading HepG2 cell model, we found that quercetin could reduce LDL induced cholesterol accumulation. Mechanistically, both mRNA and protein expression level of SREBP2 and HMGCR were down-regulated by quercetin, resulted in the regulation of cholesterol metabolism disorders. In addition, it also indicated that quercetin could inhibit cholesterol accumulation, rather than increase TG accumulation in HepG2 cells by up-regulating LXR $\beta$. Therefore, quercetin can assist HepG2 cells to remove LDL from the circulation. Although recent study suggested 


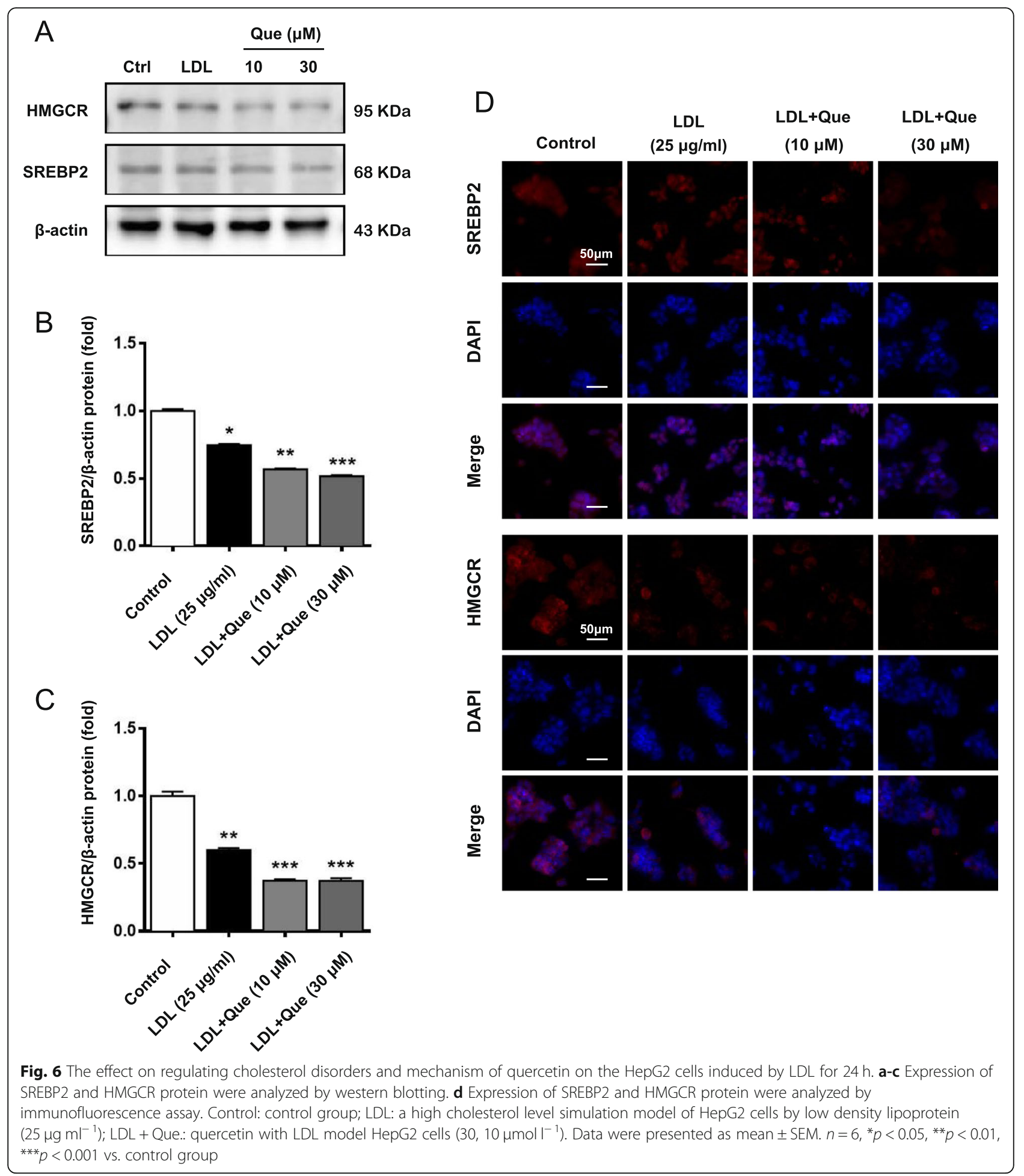

that the role in treating NAFLD has been partially elucidated as the regulation of lipid metabolism related SREBP-1c, FAS and SCD1 [14], the mechanism for regulating TC metabolism are still unclear, we speculate that it may be related to the synthesis and secretion of cholesterol regulated by SREBP2 and HMGCR.
Therefore, we have proved that MLF and quercetin can inhibit the de novo synthesis of cholesterol and promote the elimination of cholesterol, thus preventing the accumulation of cholesterol in the liver. In addition, through the decrease of LXR $\beta$ mRNA expression, it is reasonable to speculate that quercetin can inhibit the 
expression of lipid-related mRNA and protein in the liver lipid metabolism pathway, but this needs further study.

\section{Conclusion}

Our study demonstrated that MLF and quercetin could reduce the excessive cholesterol accumulation in vivo and in vitro. The cholesterol-lowering effect of MLF might attribute to its role in down-regulating the expression of SREBP2 and HMGCR, and up-regulating the expression of CYP7A1. These results suggest that quercetin, as the main metabolite of MLF, can regulate cholesterol metabolic disorders and reduce the risk of NAFLD.

\section{Supplementary information}

Supplementary information accompanies this paper at https://doi.org/10. 1186/s12906-020-03052-w

Additional file 1: Supplementary Fig. 1. Full scans of western-blot data were shown in Fig. 4. Rectangles delimit cropped areas used in the indicated panels in Fig. 4. Supplementary Fig. 2. Full scans of westernblot data were shown in Fig. 6. Rectangles delimit cropped areas used in the indicated panels in Fig. 6.

\section{Abbreviations}

BSA: bovine serum albumin; CDNA: complemental deoxyribonucleic acid; CVD: cardiovascular disease; CYP7A1: cholesterol-7a-hydroxylase; DAPI: 4',6diamidino-2-phenylindole; DMSO: dimethyl sulfoxide; FBS: fetal bovine serum; GAPDH: glyceraldehyde-3-phosphate dehydrogenase; H\&E: hematoxylin-eosin; HMGCR: HMG-CoA reductase; HPLC: high performance liquid chromatography; LDL: low density lipoprotein; LDLC: Iow density lipoprotein cholesterol; LXRa: liver $X$ receptor $a_{\text {; }}$ LXR $\beta$ : liver $X$ receptor $\beta$; MEM: Eagle's minimal essential medium; miRNA: microRNA; MLF: mulberry leaves flavonoids; NAFLD: nonalcoholic fatty liver disease; NAS: NAFLD activity score; NEAA: non-essential amino acids; OA: orotic acid; PBS: phosphate buffer saline; RT: room temperature; SR-BI: scavenger receptor class B type 1; SREBP2: sterol regulatory element binding protein 2; TC: total cholesterol; TG: triglyceride

\section{Acknowledgments}

None.

\section{Authors' contributions}

TW, YZ and ML conceived and designed the experiments. YH conducted most of experiments and analyzed the data with the help from SW, JX, QC and HY. JX, TW and ML drafted the manuscript. All authors revised and approved the final manuscript.

\section{Funding}

This work was funded by Important Drug Development Fund, Ministry of Science and Technology of China (2018ZX09735-002), National Natural Science Foundation of China (81673703) to TW. The funders had no role in study design and collection, analysis, and interpretation of data, or the decision to submit the work for publication.

\section{Availability of data and materials}

Materials used and data collected in this study are available from the corresponding author on reasonable request.

\section{Ethics approval and consent to participate}

Authors followed the Guidance Suggestions for the Care and Use of Laboratory Animals issued by the Ministry of Science and Technology of China (2006) and Science and Technological Committee and the Animal Use and Care Committee of TJUTCM (No. 201810009).

\section{Consent for publication}

All co-authors have consented for the publication of this manuscript.

\section{Competing interests}

The authors declare that they have no known competing financial interests or personal relationships that could have appeared to influence the work reported in this paper.

Received: 12 June 2020 Accepted: 11 August 2020

Published online: 17 August 2020

\section{References}

1. Rinella ME, Sanyal AJ. Management of NAFLD: a stage-based approach. Nat Rev Gastro Hepat. 2016;13(4):196-205.

2. Chacko KR, Reinus J. Extrahepatic complications of nonalcoholic fatty liver disease. Clin Liver Dis. 2016;20(2):387-401.

3. Bedogni G, Gastaldelli A, Foschi FG. Fatty liver, cardiometabolic disease and mortality. Curr Opin Lipidol. 2020;31(1):27-31.

4. Seo HS, Choi MH. Cholesterol homeostasis in cardiovascular disease and recent advances in measuring cholesterol signatures. J Steroid Biochem Mol Biol. 2015;153:72-9.

5. Karr S. Epidemiology and management of hyperlipidemia. Am J Manag Care. 2017;23(9 Suppl):S139-48

6. Arnaud C, Veillard NR, Mach F. Cholesterol-independent effects of statins in inflammation, immunomodulation and atherosclerosis. Cardiovas \& Haemat Dis. 2005:5(2):127.

7. Sorrentino S, Landmesser U. Nonlipid-lowering effects of statins. Curr Treat Options Cardiovasc Med. 2005;7(6):459-66.

8. Piglionica M, Cariello M, Moschetta A. The gut-liver axis in hepatocarcinoma: a focus on the nuclear receptor FXR and the enterokine FGF19. Curr Opin Pharmacol. 2018;43:93-8.

9. Cobbina E, Akhlaghi F. Non-alcoholic fatty liver disease (NAFLD) -pathogenesis, classification, and effect on drug metabolizing enzymes and transporters. Drug Metab Rev. 2017;49(2):197-211.

10. Al-Dabbagh B, Elhaty IA, Elhaw M, Murali C, Al Mansoori A, Awad B, Amin A Antioxidant and anticancer activities of chamomile (Matricaria recutita L.). BMC Res. Notes. 2019;12(1):3.

11. Hamza AA, Heeba GH, Elwy HM, Murali C, El-Awady R, Amin A: Molecular characterization of the grape seeds extract's effect against chemically induced liver cancer: In vivo and in vitro analyses. Sci. Rep. 2018;8(1).

12. Al-Hrout AA, Chaiboonchoe A, Khraiwesh B, Murali C, Baig B, El-Awady R, Tarazi H, Alzahmi A, Nelson DR, Greish YE et al: Safranal induces DNA double-strand breakage and ER-stress-mediated cell death in hepatocellular carcinoma cells. Sci. Rep. 2018;8(1).

13. El-Kharrag R, Amin A, Hisaindee S, Greish Y, Karam SM. Development of a therapeutic model of precancerous liver using crocin-coated magnetite nanoparticles. Int J Oncol. 2017;50(1):212-22.

14. Wang S, Chen Q, Liu M, Yu H, Xu J, Wu J, Zhang Y, Wang T. Regulation effects of rosemary (Rosmarinus officinalis Linn.) on hepatic lipid metabolism in OA induced NAFLD rats. Food Funct. 2019;10(11):7356-65.

15. Lee ES, Kwon M, Kim HM, Woo HB, Ahn CM, Chung CH. Curcumin analog CUR5-8 ameliorates nonalcoholic fatty liver disease in mice with high-fat diet-induced obesity. Metabolism. 2020;103:154015.

16. Lee $Y$, Hsu J, Lin W, Kao S, Wang C. Upregulation of caveolin-1 by mulberry leaf extract and its major components, chlorogenic acid derivatives, attenuates alcoholic steatohepatitis via inhibition of oxidative stress. Food Funct. 2017:8(1):345-97.

17. Ju W, Kwon O, Kim H, Sung G, Kim H, Kim Y. Qualitative and quantitative analysis of flavonoids from 12 species of Korean mulberry leaves. J Food Sci Technol. 2018;55(5):1789-96.

18. Song H, Lai J, Tang Q, Zheng X. Mulberry ethanol extract attenuates hepatic steatosis and insulin resistance in high-fat diet-fed mice. Nutr Res. 2016; 36(7):710-8.

19. Kim GN, Jang HD. Flavonol content in the water extract of the mulberry (Morus alba L.) leaf and their antioxidant capacities. J Food Sci. 2011;76(6): C869-73.

20. Bule M, Abdurahman A, Nikfar S, Abdollahi M, Amini M. Antidiabetic effect of quercetin: a systematic review and meta-analysis of animal studies. Food Chem Toxicol. 2019;125:494-502.

21. J H, Y F, HZ O, B Y, YX C, GX P, GY D, T W, XM G: A sensitive LC-MS/MS method for simultaneous determination of six flavonoids in rat plasma: 
application to a pharmacokinetic study of total flavonoids from mulberry leaves. J Pharmceut. Biomed. 2013;84:189-195.

22. Liu M, Pan Q, Chen Y, Yang X, Zhao B, Jia L, Zhu Y, Zhang B, Gao X, Li X, et al. Administration of Danhong Injection to diabetic $\mathrm{db} / \mathrm{db}$ mice inhibits the development of diabetic retinopathy and nephropathy. Sci Rep. 2015;5: 11219.

23. Chen Y, Liu M, Zhao T, Zhao B, Jia L, Zhu Y, Zhang B, Gao X, Li G, Li X, et al. Danhong injection inhibits the development of atherosclerosis in both Apoe(-)/(-) and Ldlr(-)/(-) mice. J Cardiovasc Pharmacol. 2014;63(5):44152.

24. Afzali A, Weiss NS, Boyko EJ, loannou GN. Association between serum uric acid level and chronic liver disease in the United States. Hepatology. 2010; 52(2):578-89.

25. Ma Y, Chen K, Lv L, Wu S, Guo Z. Ferulic acid ameliorates nonalcoholic fatty liver disease and modulates the gut microbiota composition in high-fat diet fed ApoE-/- mice. Biomed Pharmacother. 2019;113:108753.

26. Zhang Y, Liu X, Han L, Gao X, Liu E, Wang T. Regulation of lipid and glucose homeostasis by mango tree leaf extract is mediated by AMPK and PI3K/AKT signaling pathways. Food Chem. 2013;141(3):2896-905.

27. Ioannou GN. The role of cholesterol in the pathogenesis of NASH. Trends Endocrinol Metab. 2016;27(2):84-95.

28. Li T, Francl JM, Boehme S, Chiang JY. Regulation of cholesterol and bile acid homeostasis by the cholesterol 7alpha-hydroxylase/steroid response element-binding protein 2/microRNA-33a axis in mice. Hepatology. 2013; 58(3):1111-21.

29. Wouters K, van Gorp PJ, Bieghs V, Gijbels MJ, Duimel H, Lutjohann D, Kerksiek A, van Kruchten R, Maeda N, Staels B, et al. Dietary cholesterol, rather than liver steatosis, leads to hepatic inflammation in hyperlipidemic mouse models of nonalcoholic steatohepatitis. Hepatology. 2008;48(2):47486.

30. Athyros VG, Boutari C, Stavropoulos K, Anagnostis P, Imprialos KP, Doumas $M$, Karagiannis A. Statins: an under-appreciated asset for the prevention and the treatment of NAFLD or NASH and the related cardiovascular risk. Curr Vasc Pharmacol. 2018;16(3):246

31. Ann J, Eo H, Lim Y. Mulberry leaves (Morus alba L.) ameliorate obesityinduced hepatic lipogenesis, fibrosis, and oxidative stress in high-fat diet-fed mice. Genes Nutr. 2015;10(6).

32. Trimarco V, Izzo R, Stabile E, Rozza F, Santoro M, Manzi MV, Serino F, Giacomo Schiattarella G, Esposito G, Trimarco B. Effects of a new combination of Nutraceuticals with Morus alba on lipid profile, insulin sensitivity and Endotelial function in Dyslipidemic subjects. A cross-over, randomized, double-blind trial. High blood pressure Cardiovasc. Prev. 2015; 22(2):149-54.

33. Guruvaiah P, Guo H, Li D, Xie Z. Preventive effect of Flavonol derivatives abundant Sanglan tea on long-term high-fat-diet-induced obesity complications in C57BL/6 mice. Nutrients. 2018;10(9):1276.

34. Kobayashi Y, Miyazawa M, Kamei A, Abe K, Kojima T. Ameliorative effects of mulberry (Morus alba L.) leaves on hyperlipidemia in rats fed a high-fat diet: induction of fatty acid oxidation, inhibition of Lipogenesis, and suppression of oxidative stress. Biosci Biotechnol Biochem. 2010;74(12):2385-95.

35. Xu Y, Han J, Dong J, Fan X, Cai Y, Li J, Wang T, Zhou J, Shang J. Metabolomics Characterizes the Effects and Mechanisms of Quercetin in Nonalcoholic Fatty Liver Disease Development. Int J Mol Sci. 2019;20(5).

36. Yang H, Yang T, Heng C, Zhou Y, Jiang Z, Qian X, Du L, Mao S, Yin X, Lu Q Quercetin improves nonalcoholic fatty liver by ameliorating inflammation, oxidative stress, and lipid metabolism in db/db mice. Phytother Res. 2019; 33(12):3140-52.

\section{Publisher's Note}

Springer Nature remains neutral with regard to jurisdictional claims in published maps and institutional affiliations.

Ready to submit your research? Choose BMC and benefit from:

- fast, convenient online submission

- thorough peer review by experienced researchers in your field

- rapid publication on acceptance

- support for research data, including large and complex data types

- gold Open Access which fosters wider collaboration and increased citations

- maximum visibility for your research: over $100 \mathrm{M}$ website views per year

At BMC, research is always in progress.

Learn more biomedcentral.com/submissions 